\title{
Research on Science Award Judgment
}

\author{
Shang Rui \\ Science and Technology Department, Heilongjiang Bayi Agricultural University, \\ Daqing 163319, China \\ byndkjccgk@126.com
}

\begin{abstract}
At present, we level science award according to a system of norms, e.g., innovation, practicability, academic value and social value etc. However, science achievement is the combined event with multiple properties and characteristics of uncertainty. It is vital to give an objective assessment conforming to the characteristics of multiple properties about science achievement, and it is one of ensuring the accuracy of the science award judgment. Therefore, in the paper system of norms is showed by interval-valued fuzzy number and sets, and we present an interval number to scores of experts. And further study develops function for judgment the similar degree of two interval-valued fuzzy numbers and method to order interval-valued fuzzy numbers in order to make science award judgment reasonable.
\end{abstract}

Keywords: judgment; similar degree division; order; science award

\section{Introduction}

Usually, experts level science achievement with numeral scores in the process of deciding awards through discussion, such as there are ten experts scored science achievement: $91,92,88,89,90,93,89,92,90,91$. The mean data, 90.5 will be deemed to the final score generally. But we ignore lots of helpful information. Science achievement is so complex with multiple properties that information acquired is uncertain. In addition, for vagueness of human thinking, it is difficult that to describe the judgment with clear and exclusive number. So it is appropriate to show judgment with the interval-valued fuzzy number, such as the interval-valued fuzzy number [88, 92] represents the score of this science project. That is to say, the difficulty of science achievement judgment may be settled by research on sorting and order of interval-valued fuzzy number sets

The interval-valued fuzzy number, as a generalization of ordinary fuzzy number, was introduced by Dubois [1]. Recently, some important properties of interval-valued fuzzy number have been studied by [2-4], such as an interval-valued possibilistic mean and variance of fuzzy numbers, and the expected valued of fuzzy number. Later, Chun[5] defined correlation of fuzzy numbers. Moreover, the correlation of interval-valued fuzzy numbers have been more widely studied (see [6] and [7], and [8]), like expression, approximate reasoning and pattern recognition, etc. In 2001, Matarazzo introduced a way to order interval-valued fuzzy numbers. And later on Hong described distance from one interval-valued fuzzy number to another, or to interval-valued fuzzy set. Although some important properties of interval-valued fuzzy number have been studied, those are not similar degree division for interval-valued fuzzy number. Therefore, in this paper, we briefly present and discuss the similar degree of two interval-valued fuzzy numbers, then form interval-valued fuzzy numbers with same properties into interval-valued fuzzy set. At last, sort method presented in the paper decides the order of interval-valued fuzzy numbers in one set. All methods developed are tested by judgment of science award, and results show that it is reasonable and practicable. 


\section{Similar Degree of Two Interval-valued Fuzzy Numbers}

Definition 2.1: As a generalization of ordinary fuzzy number, the notion of intervalvalued fuzzy number is defined newly in the paper. In the sequel, let

$$
\left[R^{+}\right]=\left\{\left[a^{-}, a^{+}\right]: a^{-} \leq a^{+}, a^{-}, a^{+} \in R^{+}\right\}
$$

be the family of all closed positive interval-valued fuzzy number in which $\mathrm{R}$ represents the length of discussion.

Definition 2.2: Let $a=\left[a^{-}, a^{+}\right], b=\left[b^{-}, b^{+}\right]$be any two closed interval-valued fuzzy numbers in $R^{+}$, some basic operations between them are defined as follows:

$$
\begin{aligned}
& a+b=\left[a^{-}+b^{-}, a^{+}+b^{+}\right] ; \\
& a-b=\left[a^{-}-b^{-}, a^{+}-b^{+}\right] \\
& a \times b=\left[a^{-} \times b^{-}, a^{+} \times b^{+}\right] ; \\
& \lambda a=\left[\lambda a^{-}, \lambda a^{+}\right], \lambda>0 ; \\
& a \div b=\left[a^{-} \div b^{-}, a^{+} \div b^{+}\right]
\end{aligned}
$$

Definition 2.3: Let $a=\left[a^{-}, a^{+}\right], b=\left[b^{-}, b^{+}\right]$be any two closed interval-valued fuzzy numbers in $R^{+}$. Then

$$
M_{a}^{+}=\frac{1}{2}\left(a^{+}+a^{-}\right) ; M_{a}^{-}=\frac{1}{2}\left(a^{+}-a^{-}\right) ; S_{a b}=1-\frac{\left|M_{a}^{+}-M_{b}^{+}\right|}{R} .
$$

$S_{a b}$ is said to be a similar degree function of interval-valued fuzzy numbers. We can easily obtain that $0 \leq S_{a b} \leq 1$. The closer to 1 , the higher the similarity degree.

\section{Order of Interval-valued Fuzzy Numbers}

Definition 3.1: Let $a=\left[a^{-}, a^{+}\right]$be any closed interval, for $\lambda \in[0,1], F_{a}(\lambda)$ is defined as the order function of interval-valued fuzzy numbers.

$$
F_{a}(\lambda)=\left(M_{a}^{+}-M_{a}^{-}\right)+2 \lambda M_{a}^{-}
$$

Definition 3.2: Let $a=\left[a^{-}, a^{+}\right], b=\left[b^{-}, b^{+}\right]$be any two closed intervals, for $\lambda \in[0,1]$, (1) If $F_{a}(\lambda)>F_{b}(\lambda)$, then $p\{a>b\}=p\{\lambda \mid 0 \leq \lambda \leq 1\}=1$, that is $a>b$;

(2) If $F_{a}(\lambda)<F_{b}(\lambda)$, then $p\{a<b\}=p\{\lambda \mid 0 \leq \lambda \leq 1\}=1$, that is $a<b$;

(3) If $F_{a}(\lambda)=F_{b}(\lambda)$, then $p\{a=b\}=p\{\lambda \mid 0 \leq \lambda \leq 1\}=1$, that is $a=b$.

Definition 3.3: Let $a=\left[a^{-}, a^{+}\right], b=\left[b^{-}, b^{+}\right]$be any two closed intervals, for any $\lambda \in[0,1]$, then we define $\lambda_{k} \in[0,1]$ as decision level of confidence. If $\lambda_{k}>0.5$, more than half of the people will agree to order results and it is acceptable for decision makers.

(1) If $F_{a}(\lambda) \geq F_{b}(\lambda)$ and $0 \leq \lambda \leq \lambda_{k}$, then $p\{a \geq b\}=p\left\{\lambda \mid 0 \leq \lambda \leq \lambda_{k}\right\}=\lambda_{k} \times 100 \%$;

(2) If $F_{a}(\lambda) \leq F_{b}(\lambda)$ and $0 \leq \lambda \leq \lambda_{k}$, then $p\{a \leq b\}=p\left\{\lambda \mid 0 \leq \lambda \leq \lambda_{k}\right\}=\lambda_{k} \times 100 \%$.

Example: (1) Supose $a=[78,85], b=[80,90]$, calculate $F_{a}(\lambda)-F_{b}(\lambda)=-2-5 \lambda$. For any $0 \leq \lambda \leq 1$, there is $F_{a}(\lambda)-F_{b}(\lambda)<0$, so $a<b$ and $p\{a<b\}=p\{\lambda \mid 0 \leq \lambda \leq 1\}=1$. 
(2) For $a=[80,87], b=[81,84]$, calculate $F_{a}(\lambda)-F_{b}(\lambda)=-1+3 \lambda$, for any $\lambda \leq \frac{1}{3}$, $F_{a}(\lambda) \leq F_{b}(\lambda)$, so $p\{a \leq b\}=p\left\{\lambda \mid 0 \leq \lambda \leq \frac{1}{3}\right\}=\frac{1}{3}$. That is to say $a<b$ is incredible when 0.5 is the level of confidence. Then we count probability of $a>b$ as follows: $p\{a>b\}=1-p\{a \leq b\}=1-p\left\{\lambda \mid 0 \leq \lambda \leq \frac{1}{3}\right\}=\frac{2}{3}$, therefore $a>b$ is credible.

(3) Next let us consider $a=[80,87], b=[81,85]$, we can get that $F_{a}(\lambda)-F_{b}(\lambda)=-1+2 \lambda$ easily, where $\lambda_{k}=0.5$. It suggests that $a>b, a<b$ and $a=b$ have the same chance. Considering further action to order $a$ and $b$. If $a=[80,87]$ is brief expression of $a=[80,81,83,87]$ and $b=[81,85]$ is brief expression of $b=[81,83,83,85]$, restructure $a^{\prime}=[81,83]$ and $b^{\prime}=[83,83]$ by removing the maximum and minimum number. Calculate $F_{a}(\lambda)-F_{b^{\prime}}(\lambda)=-2$ by definition 3.2, definite conclusion $a<b$ can be reached.

\section{Interval-valued Fuzzy Set Judgment Model}

Set $X=\left\{x_{1}, x_{2}, \ldots, x_{n}\right\}$ be a set of properties for judgment, where $x_{i}$ is index of judgment, e.g., $x_{i}$ stands for theoretical value or application value and so on. Let $w_{i}{ }^{*}$ be effective weight of judgment, and then we have effective weight set $w^{*}=\left\{w_{1}^{*}, w_{2}{ }^{*}, \ldots, w_{n}{ }^{*}{ }^{T}\right.$. The judgments, such as 1st prize, 2nd prize, and 3rd prize form a set $Y=\left\{y_{1}, y_{2}, \ldots, y_{m}\right\}$.

We consider a interval-valued fuzzy number matrix A, where $a_{l n}=\left[a_{l n}^{-}, a_{l n}^{+}\right]$is the element in A, where expert is named by ${ }^{n}$ and attribute is called ${ }^{l}$.

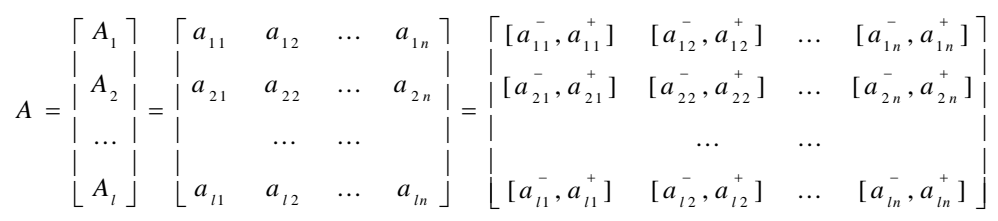

The similar degree between $a_{k i}$ and $y_{j}$ is expressed by

$$
S_{a_{k i} y_{j}}=1-\frac{M_{a_{k i}}^{+}-M_{y_{j}}^{+}}{R},
$$

where $M_{a_{k}}^{+}$and $M_{y_{j}}^{+}$is defined in previous definition 2.3 for any $k=1,2, \ldots, l$ and $j=1,2, \ldots, m$.

Then we have the similar degree $S_{A_{k} y_{j}}$ between $A_{k}$ and $y_{j}$ as follows:

$$
S_{A_{k} y_{j}}=\sum_{i=1}^{n} S_{a_{k i} y_{j}} w_{i}
$$

where $w_{i}$ is the weight of $A_{i}$. The maximum value of $S_{A_{k} y_{j}}$ be marked by $b_{j}$, therefore $A_{k}$ is judged to the kind of $y_{j}$.

Next, the order in one kind of $y_{j}$ between $A_{k}$ and $A_{j}$ is judged by $F_{A_{k}}(\lambda)$ and $F_{A_{j}}(\lambda)$, in which $F_{A_{k}}(\lambda)=\sum_{j=1}^{n} F_{a_{k j}}(\lambda), k=1,2, \ldots, l$. 
Weight vectors of attribute $X=\left\{x_{1}, x_{2}, \ldots, x_{n}\right\}$ are showed in interval-valued fuzzy numbers sometimes, so set is conformed as follows

$$
W_{1}=\left\{\left(w_{1}, w_{2}, \ldots, w_{n}\right)^{T} \mid i=1,2, \ldots, n, \sum_{i=1}^{n} w_{i}=1\right\} .
$$

Clearly, $w_{1}$ is the collection of all possible attribute's weight vectors showed in intervalvalued fuzzy numbers.

Let

$$
\begin{aligned}
& w_{i}^{{ }^{-}}=\max \left\{w_{i}^{-}, 1-\sum_{\substack{j=1 \\
j \neq i}}^{n} w_{j}^{+}\right\}, \\
& w_{i}^{+}=\min \left\{w_{i}^{+}, 1-\sum_{\substack{j=1 \\
j \neq i}}^{n} w_{j}^{-}\right\}
\end{aligned}
$$

be the left boundary and the right boundary of interval-valued fuzzy number $w_{i}^{\prime}=\left[w_{i}^{\prime-}, w_{i}^{\prime+}\right]$.

We can come to this conclusion obviously that $\left[w_{i}^{\prime-}, w_{i}^{\prime+}\right] \subseteq\left[w_{i}^{-}, w_{i}^{+}\right]$.

And let

$$
W_{2}=\left\{\left(w_{1}, w_{2}, \ldots, w_{n}\right)^{T} \mid w_{i} \in w_{i}, i=1,2, \ldots, n, \sum_{i=1}^{n} w_{i}=1\right\}
$$

be the set of $w_{i}^{\prime}$.

Theorem 4.1: $W_{1}=w_{2}$.

Proof. (1) We have $\left[w_{i}^{--}, w_{i}^{w^{\prime+}}\right] \subseteq\left[w_{i}^{-}, w_{i}^{+}\right]$, that is to say $w_{1} \supseteq W_{2}$.

(2) In the similar way, let $\left(w_{1}, w_{2}, \ldots, w_{n}\right)^{T} \in W_{1}$, then $w_{i}^{-} \leq w_{i} \leq w_{i}^{+}$. Hence $\sum_{i=1}^{n} w_{i}=1$, $w_{i}=1-\sum_{\substack{j=1 \\ j \neq i}}^{n} w_{j}$. And for $\sum_{\substack{j=1 \\ j \neq i}}^{n} w_{j}^{-} \leq \sum_{\substack{j=1 \\ j \neq i}}^{n} w_{j} \leq \sum_{\substack{j=1 \\ j \neq i}}^{n} w^{+}$, we can conclude that $1-\sum_{\substack{j=1 \\ j \neq i}}^{n} w_{j}^{+} \leq w_{i}=1-\sum_{\substack{j=1 \\ j \neq i}}^{n} w_{j} \leq 1-\sum_{\substack{j=1 \\ j \neq i}}^{n} w_{j}^{-} \quad$. Therefore $\quad w_{i} \geq \max \left\{w_{i}^{-}, 1-\sum_{\substack{j=1 \\ j \neq i}}^{n} w_{j}^{+}\right\} \quad$ and $w_{i} \leq \min \left\{w_{i}^{+}, 1-\sum_{\substack{j=1 \\ j \neq i}}^{n} w_{j}^{-}\right\}$, That is to say $w_{i} \in w_{i}^{\prime}$ and $\left(w_{1}, w_{2}, \ldots, w_{n}\right)^{T} \in W_{2}$. The same representation is $W_{1} \subseteq W_{2}$, so $W_{1}=W_{2}$.

From definition 4.1 we know ${ }^{w}$ which is the collection of all possible attribute's weight vectors showed in interval-valued fuzzy numbers is the same with $w^{\prime}=\left(w^{\prime}, w_{2}^{\prime}, \ldots, w_{n}^{\prime}\right)^{T}$, and the weight vector $w^{\prime}$ is a subset of $w$. So when we obtain the interval-valued fuzzy numbers weight vector $w$ of attributes, it is necessary to judge whether it can be compressed into the smaller interval weight vector $w^{\prime}$ or not by the formula (1).

In order to calculate the effective weight vector of attribute which corresponds to the weight vector of attribute in interval-valued fuzzy numbers, let the attribute values are clear single values about every project $A_{i}$. 
For any $w=\left(w_{1}, w_{2}, \ldots, w_{n}\right)^{T} \in W_{2}, a_{1}, a_{2}, \ldots a_{n}$ are values of project $A_{i}$ on attributes, then the global attribute value of project $A_{i}$ is showed in formula (3) by weight vector $w$ as follows:

$$
z(w)=\sum_{i=1}^{n} w_{i} x_{i}
$$

The average value of comprehensive attribute is indicated by $\hat{z}_{\left(W_{2}\right)}$, in which $W_{2}$ is the comprehensive weight vector of $A_{i}$ 's attribute, and we judge project $A_{i}$ with $z_{\left(W_{2}\right)}$.

For there are infinite weight vectors in set $w_{2}$, we put forward definition 4.1 to solve $z\left(W_{2}\right)$.

Definition 4.1: If equation $z\left(w^{*}\right)=\hat{z}\left(W_{2}\right)$ were right, $w^{*}$ would be called effective weight vector of attribute with the relevant $w$ in interval-valued fuzzy numbers, and $\hat{z}\left(W_{2}\right)$ is the comprehensive attribute value, in which $w^{*}=\left(w_{1}^{*}, w_{2}{ }^{*}, \ldots, w_{n}{ }^{*}\right)^{T} \in W_{2}$.

The effective weight vector of attribute is computed on the basis of theorem 4.2.

Theorem 4.2: Let $w^{*}=\left(w_{1}^{*}, w_{2}{ }_{2}^{*}, \ldots, w_{n}{ }_{n}\right)^{T}$ be effective weight vector of attribute corresponding with the interval weight vector $w=\left(w_{1}, w_{2}, \ldots, w_{n}\right)^{T}$.

Then

$$
\begin{aligned}
& w_{i}^{*^{*}}=\left(w_{i}^{\prime-}+\varepsilon\left(w_{i}^{+^{+}-w_{i}^{\prime}}\right)\right)^{T} \\
& \varepsilon=\frac{1-\sum_{j=1}^{n} w_{j}^{\prime-}}{\sum_{j=1}^{n}\left(w_{j}^{\prime+}-w_{j}^{\prime-}\right)}
\end{aligned}
$$

Proof. Reference [11] shows that a similar conclusion in discussing politic expected value on Interval probability, so the verification process are skipped.

\section{Science Award Judgment Case}

To take one example, the current standard of judgment is showed by $X=\left\{x_{1}, x_{2}, x_{3}, x_{4}, x_{5}\right\}$ in Daqing, where

$x_{1}$ is difficult and hot in the current international economic and social development;

$x_{2}$ is full and accurate information about research project;

$x_{3}$ is exactly and profound analysis;

$x_{4}$ is operational suggestion and measures, etc;

$x_{5}$ is high theoretical and practical value.

Let $W=\left\{w_{1}, w_{2}, w_{3}, w_{4}, w_{5}\right\}^{T}$ be attribute weight given by experts, in which

$$
\begin{aligned}
& w_{1}^{\prime}=[0.1675,0.1878] \\
& w_{2}^{\prime}=[0.1730,0.1920] \\
& w_{3}^{\prime}=[0.1505,0.1569] . \\
& w_{4}^{\prime}=[0.1414,0.1472] \\
& w_{5}=[0.3194,0.3363]
\end{aligned}
$$


The weight vector $W$ can be compressed into the smaller $W^{\prime}$ in interval-valued fuzzy numbers in accordance with formula $(1), W^{\prime}=\left\{w_{1}^{\prime}, w_{2}{ }_{2}, w_{3}{ }_{3}^{\prime}, w_{4}{ }_{4}, w_{5}\right\}^{T}$, in which

$$
\begin{aligned}
& w_{1}^{\prime}=[0.1676,0.1878] \\
& w_{2}^{\prime}=[0.1730,0.1920] \\
& w_{3}^{\prime}=[0.1505,0.1569] \\
& w_{4}^{\prime}=[0.1414,0.1472] \\
& w_{4}^{\prime}=[0.3194,0.3363]
\end{aligned}
$$

And further calculation is given for effective weight vector $W^{*}=(0.1818,0.1864,0.1550,0.1455,0.3319)$ corresponding with $W$ on the basis of formulas

\begin{tabular}{|c|c|c|c|c|c|}
\hline & $x_{1}$ & $x_{2}$ & $x_{3}$ & $x_{4}$ & $x_{5}$ \\
\hline$A_{1}$ & $\begin{array}{c}{[90,} \\
95]\end{array}$ & $\begin{array}{c}{[92,} \\
94]\end{array}$ & $\begin{array}{c}{[89,} \\
92]\end{array}$ & $\begin{array}{c}{[90,} \\
94]\end{array}$ & $\begin{array}{c}{[80,} \\
92]\end{array}$ \\
\hline$A_{2}$ & $\begin{array}{c}{[82,} \\
83]\end{array}$ & $\begin{array}{c}{[80,} \\
84]\end{array}$ & $\begin{array}{c}{[85,} \\
86]\end{array}$ & $\begin{array}{c}{[83,} \\
85]\end{array}$ & $\begin{array}{c}{[82,} \\
89]\end{array}$ \\
\hline$A_{3}$ & $\begin{array}{c}{[92,} \\
93]\end{array}$ & $\begin{array}{c}{[90,} \\
95]\end{array}$ & $\begin{array}{c}{[92,} \\
93]\end{array}$ & $\begin{array}{c}{[91,} \\
95]\end{array}$ & $\begin{array}{c}{[89,} \\
92]\end{array}$ \\
\hline$A_{4}$ & $\begin{array}{c}{[70,} \\
72]\end{array}$ & $\begin{array}{c}{[72,} \\
73]\end{array}$ & $\begin{array}{c}{[71,} \\
72]\end{array}$ & $\begin{array}{c}{[73,} \\
75]\end{array}$ & $\begin{array}{c}{[73,} \\
74]\end{array}$ \\
\hline$A_{5}$ & $\begin{array}{c}{[60,} \\
68]\end{array}$ & $\begin{array}{c}{[62,} \\
69]\end{array}$ & $\begin{array}{c}{[60,} \\
62]\end{array}$ & $\begin{array}{c}{[61,} \\
63]\end{array}$ & $\begin{array}{l}{[61,} \\
69]\end{array}$ \\
\hline$A_{6}$ & $\begin{array}{c}{[75,} \\
76]\end{array}$ & $\begin{array}{c}{[73,} \\
77]\end{array}$ & $\begin{array}{c}{[72,} \\
75]\end{array}$ & $\begin{array}{c}{[71,} \\
73]\end{array}$ & $\begin{array}{c}{[70,} \\
71]\end{array}$ \\
\hline
\end{tabular}
(4) and (5) in theorem 4.2.

There are 10 experts to order 6 projects. Experts score to attributes in interval-valued fuzzy numbers for each project as Table 1.

Table 1. Interval Scores of Scientific Achievements

In the same way, judgment order is $\{1$ st prize, 2 nd prize, 3 rd prize, honorable mention, no prize $\}$ presented by interval-valued fuzzy set, as

$$
Y=\left\{y_{1}, y_{2}, y_{3}, y_{4}, y_{5}\right\}=\{[90,100],[80,89],[70,79],[60,69],[0,59]\} \text {. }
$$

Project $A_{1}$ and judgment order $y_{1}$ similarity can be calculate by function $S_{A_{1} y_{1}}=\sum_{i=1}^{5} s_{a_{1 i} y_{1}} w_{i}^{*}$, therefore $S_{A_{1} y_{1}}=0.9515, S_{A_{1} y_{2}}=0.9435, S_{A_{1} y_{3}}=0.9135, S_{A_{1} y_{4}}=0.7435$, and $S_{A_{1} y_{5}}=0.3935$. From results, we could conclude that $A_{1}$ gets the first prize for the highest similarity to $y_{1}$.

Further calculations, degree of similarity about projects and judgment orders are listed in Table 2 
Table 2. Similarity between Projects and Judgment Orders

\begin{tabular}{rrrrrrrr}
\hline$S$ & $A_{1}$ & $A_{2}$ & $A_{3}$ & $A_{4}$ & $A_{5}$ & $A_{6}$ \\
\hline$y_{1}$ & 0.9493 & 0.9282 & 0.9298 & 0.7767 & 0.6890 & 0.7707 \\
& & & & & & \\
$y_{2}$ & 0.9469 & 0.9867 & 0.9297 & 0.8818 & 0.7940 & 0.9003 \\
& & & & & & \\
$y_{3}$ & 0.8468 & 0.9047 & 0.8298 & 0.9818 & 0.8941 & 0.9808 \\
& & & & & & \\
$y_{4}$ & 0.7468 & 0.8415 & 0.7297 & 0.9193 & 0.9871 & 0.9144 \\
& & & & & & \\
$y_{5}$ & 0.3966 & 0.5809 & 0.3795 & 0.5691 & 0.6562 & 0.5847
\end{tabular}

From Table 2, projects $A_{1}$ and $A_{3}$ win the first prize, the project $A_{2}$ wins the second prize, projects $A_{4}$ and $A_{6}$ win the third prize, the project $A_{5}$ is honorable mention.

The order between $A_{1}, A_{3}$, and between $A_{4}, A_{6}$ in the same award is calculated in accordance with definition 3.2 and definition 3.3.

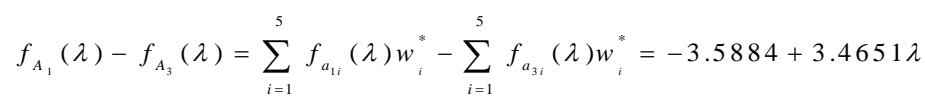

For any $\lambda \in[0,1]$, there is $f_{A_{1}}(\lambda)-f_{A_{3}}(\lambda)<0$, and that is to say $A_{3}$ is in front of $A_{1}$.

Calculated by $f_{A_{4}}(\lambda)-f_{A_{6}}(\lambda)=0.0363-0.6874 \lambda=0, \lambda_{k}=0.0528$. Thus, $P\left\{A_{4}<A_{6}\right\}=P\left\{\lambda \geq \lambda_{k}\right\}=99.472 \%$.

That is to say $A_{6}$ is in front of $A_{4}$.

Therefore the total order is $A_{3}, A_{1}, A_{2}, A_{6}, A_{4}$.

\section{Conclusion Remarks}

In this paper, the definition, some basic operations, similar degree and order in intervalvalued fuzzy numbers have been proposed and investigated. We extend the works to interval-valued fuzzy sets in positive real domain. Then, we present functions to calculate similar degree and order of interval-valued fuzzy numbers. Finally, one example has been given. Judgment order and standard of science award are expressed in interval-valued fuzzy numbers, and experts score to attributes in interval-valued fuzzy numbers for each project, calculating according to similar degree and order function, then we get science award ranking order.

\section{Acknowledgements}

This research was supported by the Science and Technology Research Project of Daqing of China (Project name: Network Innovation Research on Scientific Project Management in Daqing) and the Science and Technology Research Project of Heilongjiang Land Reclamation Bureau of China (Project name: Software Construction on Network for 
Scientific Project Plan and Process Management of Heilongiang Land Reclamation Bureau), all support is gratefully acknowledged.

\section{References}

[1] D. Dubois and H. Prade, "The mean value of a fuzzy number", Fuzzy Sets and Systems, (1987).

[2] S. Heilpern, "The expected value of a fuzzy number", Fuzzy Sets and Systems, (1992).

[3] C. Carlsson, "On possibilistic mean value and variance of fuzzy numbers", Fuzzy Sets and Systems, (2001).

[4] R. Fuller and P. Majlender, "On weighted possibilistic mean and variance of fuzzy numbers", Fuzzy Sets and Systems, (2003).

[5] C. H. Yu, Correlation of fuzzy numbers, Fuzzy Sets and Systems, (1993).

[6] D. H. Hong, "A note on the correlation of fuzzy numbers", Fuzzy Sets and Systems, (1996).

[7] D. H. Hong, "Correlation of intuitionistic fuzzy sets in probability spaces", Fuzzy Sets and Systems, (1995).

[8] D. H. HONG, "A note on correlation of interval-valued intuitive fuzzy sets", Fuzzy Sets and Systems, (1998).

[9] B. Matarazzo, "New approaches for the comparison of L-R fuzzy numbers: a theoretical and operational analysis", Fuzzy Sets and Systems, (2001).

[10] D. H. Hong, "Some algebraic properties and a distance measure for interval-valued fuzzy numbers", Journal of Information Science, (2002).

[11] R. R. Yager and V. Kreinovich, "Decision Making under Interval Probabilities", Iinternationl Journal of Approximate Reasoning, (1999).

\section{Author}

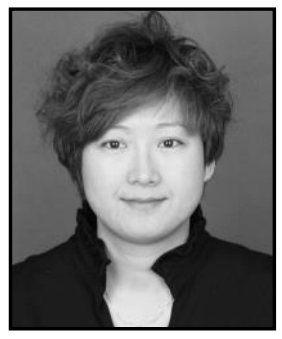

\section{Shang Rui}

Author's profile: Shang Rui (1979-), female, Mongolian nationality, native place:Heilongjiang. Researcher, Bachelor degree. Tel: 13936929898, E-mail: byndkjccgk@126.com 